\title{
IFL-FOLFIRI Regimen
}

National Cancer Institute

\section{Source}

National Cancer Institute. IFL-FOLFIRI Regimen. NCI Thesaurus. Code C64191.

A regimen consisting of irinotecan, infusional fluorouracil and leucovorin used for the treatment of advanced stage colorectal cancer. This regimen differs from the Douillard regimen with regards to the dose of fluorouracil administered. 